\title{
Efficacy of a Single Oral Administration of Afoxolaner Alone or in Combination with Milbemycin Oxime against Ixodes hexagonus Ticks in Dogs
}

\author{
W. Lebon'1, M. Servonnet ${ }^{2}$, D. Larsen ${ }^{3}$, P. Dumont ${ }^{1}$, F. Beugnet ${ }^{2}$ \\ ${ }^{1}$ Boehringer Ingelheim Animal Health, Saint-Vulbas, France \\ ${ }^{2}$ Boehringer Ingelheim Animal Health, Lyon, France \\ ${ }^{3}$ Boehringer Ingelheim Animal Health, Duluth, GA, USA \\ Email: *marielle.servonnet@boehringer-ingelheim.com
}

How to cite this paper: Lebon, W., Servonnet, M., Larsen, D., Dumont, P. and Beugnet, F. (2019) Efficacy of a Single Oral Administration of Afoxolaner Alone or in Combination with Milbemycin Oxime against Ixodes hexagonus Ticks in Dogs. Open Journal of Veterinary Medicine, 9, 45-54.

https://doi.org/10.4236/ojvm.2019.94004

Received: April 12, 2019

Accepted: April 27, 2019

Published: April 30, 2019

Copyright (c) 2019 by author(s) and Scientific Research Publishing Inc. This work is licensed under the Creative Commons Attribution International License (CC BY 4.0).

http://creativecommons.org/licenses/by/4.0/

\section{Open Access}

\begin{abstract}
The efficacy of afoxolaner (NexGard ${ }^{\circledR}$ and NexGard Spectra ${ }^{\circledR}$, Boehringer-Ingelheim), administered once orally at the minimum recommended dose, was assessed in dogs experimentally infested with Ixodes hexagonus ticks. The study was a blinded, negative controlled clinical efficacy study using a randomized block design. Twenty-four Beagle dogs, 12 females and 12 males were enrolled. Dogs were randomly allocated either to the negative control group, or to one of the two treated groups. Infestations were performed with 50 adult $I$. hexagonus ticks on Days-2, 7 and 28. On Day 0, dogs in groups 2 and 3 were treated with NexGard ${ }^{\circledR}$ (afoxolaner) or NexGard Spectra ${ }^{\circledR}$ (afoxolaner + milbemycin oxime), respectively. Tick counts were conducted 48 hours after treatment (Day 2) and 48 hours after each subsequent infestation (Days 9 and 30). In both treated groups, afoxolaner was $100 \%$ effective against existing infestations $(\mathrm{p}<0.0001)$. Regarding the re-infestations, the efficacy of afoxolaner was $100 \%$ on Day 9 for both products, $96.5 \%$ and $100 \%$ on Day 30 for NexGard ${ }^{\circledR}$ and NexGard Spectra ${ }^{\circledR}$ respectively. NexGard ${ }^{\circledR}$ and NexGard Spectra ${ }^{\circledR}$ chewable tablets administered once orally at the minimum recommended dose were highly effective against $I$. hexagonus infestations for the 4 weeks duration of the study.
\end{abstract}

\section{Keywords}

Dog, Afoxolaner, Tick, Ixodes hexagonus

\section{Introduction}

Ticks are quite common ectoparasites, with over 800 species described world- 
wide, and more than twelve different species are recognized as parasites of dogs in Europe. The clinical significance of tick infestation in dogs is not only based on direct pathogenic effects (e.g. skin reactions and anemia in case of heavy burdens of ticks), but mainly linked to them as potential vectors. Transmitted pathogens include viral, bacterial, and protozoan agents, among which some may result in severe or life-threatening diseases [1] and/or are zoonotic.

Widespread and endemic in Europe, Ixodes species are known vectors of several pathogens of animal and human significance (e.g. Borrelia burgdorferi, Anaplasma phagocytophilum, Tick-Borne Encephalitis virus...).

The most common tick species parasitizing dogs in Western, Central and Eastern Europe is Ixodes ricinus, also called the wood tick. Other Ixodes species such as Ixodes hexagonus, and Ixodes canisuga, may be found in dogs but their prevalence is lower [2]-[9].

The so-called Hedgehog tick, Ixodes hexagonus, is a nidicolous tick which has been described in many parts of Europe such as UK and Ireland [3] [8], France [4] [10], Belgium [9], Netherlands [6], Germany [11], Iceland [12], Poland [13], Switzerland [14], Italy [15], and Spain [16]. While less prevalent than Ixodes ricinus, I. hexagonus seems to be the second most common tick found on dogs in some parts of Western Europe. It has been reported to represent 1.2\% to 5.6\% of ticks collected on dogs examined in recent studies [14] [15]. Other studies have shown that amongst tick-infested dogs, $8.8 \%$ to $22.6 \%$ of them carry I. hexagonus [8] [9] [11], even if in low numbers.

While Ixodes hexagonus is mainly an endophilic tick species, typically living in its host's nest, it is able to infest and feed not only on medium-sized wild carnivores such as foxes and mustelids, but also on domestic mammals-cats or dogs, and may attach to humans. It usually does not feed on birds. The hedgehog tick may be observed in urban and suburban areas, with urban parks and gardens potentially supporting significant populations of this tick species [3].

Ixodes hexagonus is a proven vector of the spirochete Borrelia burgdorferi, the causative agent of Lyme disease [17] [18], and is suspected to be the vector of Theileria annae (syn. Babesia microti-like-Babesia vulpes [19]) in dogs in Spain [16]. It has also been found to harbour Rickettsia helvetica, Anaplasma phagocytophilum, the Tick-Borne Encephalitis (TBE) virus, and its capacity as a vector has been suggested for all of these pathogens [6] [20] [21].

Previous experimental and field studies have demonstrated that afoxolaner is highly effective against existing and new infestations of several tick species in dogs for at least 4 weeks [22]-[29], but its efficacy against Ixodes hexagonus ticks has not been examined.

The objective of the present laboratory study was to evaluate the efficacy of afoxolaner in both marketed formulations (NexGard ${ }^{\circledR}$-afoxolaner and NexGard Spectra ${ }^{\circledR}$-afoxolaner plus milbemycin oxime-chewable tablets), administered once orally at the minimum recommended dose against induced Ixodes hexagonus infestations for a period of four weeks. 


\section{Materials and Methods}

The general study design followed the Committee For Medicinal Products for Veterinary Use (CVMP), "Guideline for the testing and evaluation of the efficacy of antiparasitic substances for the treatment and prevention of tick and flea infestation in dogs and cats", EMEA/CVMP/EWP/005/2000-Rev.3, as well as the World Association for the Advancement of Veterinary Parasitology (WAAVP) second edition guidelines for evaluating the efficacy of parasiticides for the treatment, prevention and control of flea and tick infestation on dogs and cats [30]. The EMA guideline recommends the sample size to get statistically significant results (at least 6 animals per treatment/control group), as well as the schedule of operations regarding the time points for treatment and infestations to assess both curative and persistent efficacy.

The study was blinded-staff members involved in data collection and analysis or health observations had no information about the dogs' group allocationand was conducted in accordance with the Good Clinical Practice Guideline (VICH GL9).

\subsection{Animals}

The sample size was defined according to guideline recommendations in order to get 3 groups of 8 dogs: twenty-four purpose-bred Beagle dogs, 12 females and 12 males, aged 8 to 12 months and weighing 9.1 to $14.5 \mathrm{~kg}$ were enrolled.

Acclimation of the animals to the study conditions began 8 days prior to treatment (Day-8). Each dog underwent a physical examination by a veterinarian, on the first day of acclimation, and all dogs were confirmed in good health. They were fed a standard commercially available animal food. Water was available ad libitum.

No animal had been treated with any ectoparasiticide compound for three months before acclimation started.

\subsection{Allocation to Treatment Groups}

The study followed a randomized block design based on pre-treatment counts of live attached female ticks. A pre-treatment infestation and count was used to form blocks. The twenty four dogs were infested on Day-7 and tick counts were performed on Day-5. Dogs were ranked by decreasing live attached female tick count (dogs with the same tick count were ranked by decreasing identification numbers), and eight blocks of three dogs each were formed. The three dogs with the highest tick count formed Block 1; the next three highest tick counts formed Block 2, and so on, until all dogs were allocated.

Within blocks, dogs were then randomly allocated to one of the three treatment groups, using the PLAN procedure of SAS (version 9.4): Group 1, untreated control, Group 2, NexGard ${ }^{\circledR}$ (afoxolaner), and Group 3, NexGard Spectra $^{\circledR}$ (afoxolaner + milbemycin oxime).

Twenty four dogs were initially selected for the study, but one female dog 
from the NexGard Spectra treated group was excluded on Day-1 due to potential pregnancy.

Animals were group-housed in cages by treatment group and by sex throughout the animal phase except on days between tick infestations and counts where the animals were kept individually.

\subsection{Infestations, Treatment, and Tick Counts}

Treatments were administered orally, once, on Day 0. Starting two days before treatment (Day-2) all dogs were infested with 50 unfed adult (40 females +10 males) Ixodes hexagonus ticks, and new infestations were conducted on Days 7 and 28. Tick counts were performed at $\sim 48$ hours post-treatment (Day 2) and at $\sim 48$ hours following each post-treatment infestation (Days 9 and 30). For each tick infestation, the dogs were sedated for at least 30 minutes, using intramuscular (IM) or intravenous (IV) injection of medetomidine and ketamine, to allow ticks to crawl and select an attachment site. Sedations were reversed using IM injection of atipamezole.

Specific pathogen free Ixodes hexagonus ticks were provided by Utrecht Centre for Tick-borne Diseases (Faculty of Veterinary Medicine, the Netherlands) and by Biology Centre ASCR (Ceske Budejovice, Czech Republic). Ticks sourced from Utrecht center for Tick-borne diseases were used for the allocation of dogs to treatment groups (infestation on Day-7) and to infest dogs of the 3 groups on Day-2 and on Day 7, while ticks sourced from Biology Centre ASCR were used to infest dogs of the three groups on Day 28.

Group 1 dogs remained untreated while dogs from Groups 2 and 3 were treated once orally with NexGard ${ }^{\circledR}$ or NexGard Spectra ${ }^{\circledR}$ respectively. Chewables were chosen or combined as appropriate in order to dose the dogs, as close as possible, to the minimum effective dose of $2.5 \mathrm{mg}$ afoxolaner per $\mathrm{kg}$ body weight.

Dogs were observed hourly for four hours post-treatment for adverse reaction to treatment. No drug was lost and no dog vomited.

On Day 2 ( $\sim 48$ hours after treatment), all ticks were removed and counted to evaluate the curative/immediate efficacy of afoxolaner for both products.

Subsequent tick removals and counts were made on Days 9, and 30 (each time, $\sim 48$ hours after infestation) to assess the persistent/sustained efficacy of the tested products.

In order to perform the tick counts, dogs were sedated using the same procedure as described for the infestation phases, and the whole body of each dog was examined for ticks. Attached ticks were removed using a tick extractor, and each tick was observed for signs of viability or mortality. The numbers of live attached, live free, dead attached, and dead free ticks was recorded. As male Ixodes ticks do not attach and feed on blood, the efficacy analysis was therefore based on the counts of attached female ticks.

\subsection{Data Analysis}

To evaluate the afoxolaner curative-immediate/therapeutic efficacy (efficacy 
against Day-2 Ixodes hexagonus infestations) and its persistent-sustained/ preventive efficacy (efficacy against Day 7 and Day 28 infestations), arithmetic means of the live attached female tick counts were calculated by group at each time point. Percent effectiveness for each treated group was calculated using the formula:

$$
[(\mathrm{C}-\mathrm{T}) / \mathrm{C}] \times 100
$$

where $\mathrm{C}=$ arithmetic mean for the control group and $\mathrm{T}=$ arithmetic mean for the treated group.

To compare the groups, the natural logarithm of (counts +1 ) was analyzed. The log-counts of each treated group was compared to the log-counts of the control group using an F-test adjusted for the allocation blocks used to randomize the animals to the treatment groups at each time point separately. The MIXED procedure in SAS Version 9.4 was used for the analysis, with the group listed as a fixed effect and the allocation blocks listed as a random effect. All statistical comparisons were two-sided at the 0.05 significance level.

\section{Results}

\subsection{Health Observations}

The animals were observed at least once daily for general health over the whole duration of the experimental phase. Following treatment, dogs were also observed hourly for 4 hours for adverse reactions to treatment.

On Day 5, one dog in Group 3 (NexGard Spectra ${ }^{\circledR}$ treated) engaged in an altercation with the two other dogs in the same cage and received a traumatic injury in the area of the cervical spine. This dog showed neurological signs, which was attributed to this injury. The animal was euthanized per the veterinarian recommendation, and the necropsy confirmed traumatic lesions in the cervical spine.

There were no adverse events related to treatment observed throughout the study, including during hourly observations conducted for 4 hours immediately after treatment.

\subsection{Efficacy}

Individual retention rates of live attached female ticks on the control animals ranged from $30.0 \%$ to $87.5 \%$ (Mean: $54.1 \%$ - 66.3\%), demonstrating adequate infestation conditions with a vigorous tick population.

The percentage of efficacy of afoxolaner, using arithmetic means, in NexGard $^{\circledR}$ group (Group 2) compared to the untreated control group was $100 \%$, $100 \%$ and $96.7 \%$ on Days 2, 9 and 30, respectively. The percent efficacy of afoxolaner, using arithmetic means, in NexGard Spectra ${ }^{\circledR}$ group (Group 3) compared to the untreated control group was $100 \%$ at all post-treatment counts. There were significant differences in the population means between the treated groups and control group at all time points ( $\mathrm{p}<0.0001)$.

The analysis of the results is summarized in Table 1. 
Table 1. Summary of analysis results of live attached female tick counts.

\begin{tabular}{ccccc}
\hline Study Day & $\begin{array}{c}\text { Group 1 } \\
\mathrm{AM}^{2}\left(\mathrm{n}^{3}\right)\end{array}$ & $\begin{array}{c}\text { Group 2 } \\
\mathrm{AM}^{2}\left(\mathbf{n}^{3}\right)\end{array}$ & \% Efficacy & p-Value $^{5}$ \\
\hline 2 & $24.5(8)$ & $0(8)$ & 100 & $<0.0001$ \\
9 & $21.6(8)$ & $0(8)$ & 100 & $<0.0001$ \\
30 & $26.5(8)$ & $0.9(8)$ & 96.7 & $<0.0001$ \\
\hline \multirow{2}{*}{ Study Day } & $\mathrm{Group} \mathrm{1}^{1}$ & $\mathrm{Group} \mathrm{3}^{1}$ & \% Efficacy & p-Value $^{4}$ \\
\hline 2 & $\mathrm{AM}^{2}\left(\mathrm{n}^{3}\right)$ & $\mathrm{AM}^{2}\left(\mathrm{n}^{3}\right)$ & 100 & $<0.0001$ \\
9 & $24.5(8)$ & $0(7)$ & 100 & $<0.0001$ \\
30 & $21.6(8)$ & $0(6)$ & 100 & $<0.0001$ \\
\hline
\end{tabular}

${ }^{1}$ Group $1=$ Untreated Control; Group $2=$ NexGard ${ }^{\circledR}$; Group $3=$ NexGard Spectra ${ }^{\circledR} ;{ }^{2} \mathrm{AM}=$ tick arithmetic mean; ${ }^{3} \mathrm{n}=$ number of animals; ${ }^{4}$ Percent efficacy $=[(\mathrm{C}-\mathrm{T}) / \mathrm{C}] \times 100$, where $\mathrm{C}$ and $\mathrm{T}$ are arithmetic means of Group 1 and Group 2 counts, or arithmetic means of Group 1 and Group 3 counts respectively; ${ }^{5}$ p-value $=$ two-sided probability value from analysis of variance on log-counts of Group 2 and Group 1 or log-counts of Group 3 and Group 1.

\section{Discussion and Conclusion}

This experimental dose confirmation study was conducted to assess the efficacy of afoxolaner (NexGard ${ }^{\circledR}$ or NexGard Spectra ${ }^{\circledR}$ chewables) against Ixodes hexagonus, commonly called the hedgehog tick.

NexGard $^{\circledR}$ is an afoxolaner-based palatable formulation marketed for the monthly treatment of fleas, ticks, demodicosis and sarcoptic mange in dogs. In the USA, NexGard ${ }^{\circledR}$ is also indicated for the prevention of Borrelia burgdorferi infections as a direct result of killing Ixodes scapularis vector ticks. NexGard Spectra ${ }^{\circledR}$ chewables for dogs have been developed as a convenient option for pet owners and veterinarians to treat and control both ecto and endoparasitic infections in dogs using a combination of afoxolaner and milbemycin oxime in a single chewable tablet.

Milbemycin oxime administered to dogs orally is used in several marketed formulations against adult intestinal nematode infections and to prevent heartworm disease. Given orally at a dose of $0.5 \mathrm{mg}$ per kg body weight, this macrocyclic lactone does not demonstrate any activity against fleas and ticks in dogs, as published by Snyder and Wiseman in 2012 [31]; and as shown in some studies conducted during NexGard Spectra ${ }^{\circledR}$ development (Merial, EMA registration dossier).

To assess afoxolaner's efficacy against Ixodes hexagonus, three groups of dogs were used. Two groups of dogs were treated with afoxolaner alone (NexGard ${ }^{\circledR}$ ) or combined with milbemycin oxime (NexGard Spectra $\left.{ }^{\circledR}\right)$, both at a dose as close as possible to the minimum effective dose of $2.5 \mathrm{mg} / \mathrm{kg}$ afoxolaner. The third untreated group served as the negative control group to assess efficacy for both products.

In this study, tick challenges were not performed weekly, but on three occasions (Days-2, 7 and 28). The limited number of infestations nevertheless allowed the evaluation of afoxolaner's curative efficacy against existing ticks, as 
well as its effectiveness against re-infestations both at the beginning and at the end of the month following treatment.

Despite the loss of two dogs in the NexGard Spectra ${ }^{\circledR}$ treated group due to reasons unrelated to treatment, this blinded, randomized, negative controlled clinical efficacy study met the requirements of international guidelines for ectoparasiticide efficacy testing in terms of sample size (at least 6 dogs per group), design and adequate strength of the tick challenge to provide statistically significant results.

The efficacy of a single treatment with afoxolaner, either alone or combined with milbemycin oxime resulted in $100 \%$ curative/immediate efficacy against $I$. hexagonus pre-existing infestations.

Afoxolaner in both products was also highly effective ( $96.7 \%$ to $100 \%$ efficacy) against re-infestations for at least 28 days after treatment.

The similar results obtained for afoxolaner in both formulations confirm the clinical equivalence of the two products regarding their efficacy against $I$. hexagonus ticks, as it has been previously demonstrated against Dermacentor reticulatus ticks [26].

These results are consistent with previous experimental efficacy data published for afoxolaner against several other Ixodes species, e.g. Ixodes ricinus (98.5\% $100 \%$ efficacy for 35 days) [22], Ixodes scapularis (94.2\% - 100\% efficacy for 28 days) [24] and Ixodes holocyclus (97.7\% - 100\% efficacy for 35 days) [28].

Ixodes hexagonus ticks thus seem to show the same level of susceptibility to afoxolaner as other Ixodes species.

The safety and efficacy of afoxolaner alone or in combination with milbemycin oxime observed in the present experimental study are also in accordance with the results of the pivotal multicentric field trials conducted in Europe for both products. In these 30-day field studies, treatment with NexGard ${ }^{\circledR}$ (afoxolaner) and NexGard Spectra ${ }^{\circledR}$ (afoxolaner plus milbemycin oxime) chewables consistently demonstrated $>98 \%$ and $>95 \%$ efficacy respectively, against natural challenges with ticks including Dermacentor reticulatus, Ixodes ricinus and Rhipicephalus sanguineus [32], and Haemaphysalis concinna, D. reticulatus, I. hexagonus, I. ricinus, and $R$. sanguineus [27] [33].

In conclusion, afoxolaner administered once orally at the minimum recommended dose in NexGard ${ }^{\circledR}$ or in NexGard Spectra ${ }^{\circledR}$ chewables is safe and highly effective against both existing and new I. hexagonus infestations in dogs for 4 weeks.

\section{Acknowledgements}

The authors would like to thank all the study personnel at CRSV for their technical assistance and Stephen Yoon for statistical analysis.

\section{Funding}

This clinical study was funded by Boehringer Ingelheim Animal Health. Wilfried 
Lebon, Marielle Servonnet, Diane Larsen, Pascal Dumont and Frederic Beugnet are employees of Boehringer Ingelheim Animal Health.

\section{Conflicts of Interest}

The authors declare no conflicts of interest regarding the publication of this paper.

\section{References}

[1] Dryden, M.W. and Payne, P.A. (2004) Biology and Control of Ticks Infesting Dogs and Cats in North America. Veterinary Therapeutics, 5, 139-154.

[2] Jaenson, T.G., Tälleklint, L., Lundqvist, L., Olsen, B., Chirico, J. and Mejlon, H. (1994) Geographical Distribution, Host Associations, and Vector Roles of Ticks (Acari: Ixodidae, Argasidae) in Sweden. Journal of Medical Entomology, 31, 240-256. https://doi.org/10.1093/jmedent/31.2.240

[3] Ogden, N.H., Cripps, P., Davison, C.C., Owen, G., Parry, J.M., Timms, B.J. and Forbes, A.B. (2000) The Ixodid Tick Species Attaching to Domestic Dogs and Cats in Great Britain and Ireland. Medical and Veterinary Entomology, 14, 332-338. https://doi.org/10.1046/j.1365-2915.2000.00244.x

[4] Marotel, M.L.S. (2006) Tiques des carnivores domestiques en régions Rhône-Alpes, Auvergne, Limousin, Midi-Pyrénées, Aquitaine. Enquête de Juin à Décembre 2004. Thèse Doct Vét, Ecole Nat Vét Toulouse.

[5] Földvári, G. and Farkas, R. (2005) Ixodid Tick Species Attaching to Dogs in Hungary. Veterinary Parasitology, 129, 125-131.

https://doi.org/10.1016/j.vetpar.2004.11.032

[6] Nijhof, A.M., Bodaan, C., Postigo, M., Nieuwenhuijs, H., Opsteegh, M., Franssen, L., Jebbink, F. and Jongejan, F. (2007) Ticks and Associated Pathogens Collected from Domestic Animals in the Netherlands. Vector-Borne and Zoonotic Diseases, 7, 585-596. https://doi.org/10.1089/vbz.2007.0130

[7] Omeragić, J. (2011) Ixodid Ticks in Bosnia and Herzegovina. Experimental and Applied Acarology, 53, 301-309. https://doi.org/10.1007/s10493-010-9402-8

[8] Smith, F.D., Ballantyne, R., Morgan, E.R. and Wall, R. (2011) Prevalence, Distribution and Risk Associated with Tick Infestation of Dogs in Great Britain. Medical and Veterinary Entomology, 25, 377-384.

https://doi.org/10.1111/j.1365-2915.2011.00954.x

[9] Claerebout, E., Losson, B., Cochez, C., Casaert, S., Dalemans, A.C., De Cat, A., Madder, M., Saegerman, C., Heyman, P. and Lempereur, L. (2013) Ticks and Associated Pathogens Collected from Dogs and Cats in Belgium. Parasites and Vectors, 6, 183. https://doi.org/10.1186/1756-3305-6-183

[10] Pichot, J., Gilot, B., Almire, N., Polette, K. and Degeilh, B. (1997) Ixodes Populations (I. ricinus Linné, 1758, I. hexagonus Leach, 1815) in the City of Lyon (France) and Its Outskirts: Preliminary Results. Parasite, 4, 167-171. https://doi.org/10.1051/parasite/1997042167

[11] Beck, S., Schreiber, C., Schein, E., Krücken, J., Baldermann, C., Pachnicke, S., von Samson-Himmelstjerna, G. and Kohn, B. (2014) Tick Infestation and Prophylaxis of Dogs in Northeastern Germany: A Prospective Study. Ticks and Tick-Borne Diseases, 5, 336-342. https://doi.org/10.1016/j.ttbdis.2013.12.009

[12] Richter, S.H., Eydal, M., Skírnisson, K. and Ólafsson, E. (2013) Tick Species (Ixodida) Identified in Iceland. Icelandic Agricultural Sciences, 26, 3-10. 
[13] Król, N., Obiegala, A., Pfeffer, M., Lonc, E. and Kiewr, D. (2016) Detection of Selected Pathogens in Ticks Collected from Cats and Dogs in the Wrocław Agglomeration, South-West Poland. Parasites and Vectors, 9, 351. https://doi.org/10.1186/s13071-016-1632-0

[14] Eichenberger, R.M., Desplazes, P. and Mathis, A. (2015) Ticks on Dogs and Cats: A Pet Owner-Based Survey in a Rural Town in Northeastern Switzerland. Ticks and Tick-Borne Diseases, 6, 267-271. https://doi.org/10.1016/j.ttbdis.2015.01.007

[15] Maurelli, M.P., Pepe, P., Colombo, L., Armstrong, R., Battisti, E., Morgoglione, M.E., Counturis, D., Rinaldi, L., Cringoli, G., Ferroglio, E. and Zanet, S. (2018) A National Survey of Ixodidae Ticks on Privately Owned Dogs in Italy. Parasites and Vectors, 11, 420. https://doi.org/10.1186/s13071-018-2994-2

[16] Camacho, A.T., Pallas, E., Gestal, J.J., Guitián, F.J., Olmeda, A.S., Telford, S.R. and Spielman, A. (2003) Ixodes hexagonus Is the Main Candidate as Vector of Theileria annae in Northwest Spain. Veterinary Parasitology, 112, 157-163.

https://doi.org/10.1016/S0304-4017(02)00417-X

[17] Gern, L., Toutoungi, L.N., Hu, C.M. and Aeschlimann, A. (1991) Ixodes (Pholeoixodes) hexagonus, an Efficient Vector of Borrelia burgdorferi in the Laboratory. Medical and Veterinary Parasitology, 5, 431-435. https://doi.org/10.1111/j.1365-2915.1991.tb00571.x

[18] Piesman, J. and Gern, L. (2004) Lyme borreliosis in Europe and North America. Parasitology, 129, S191-S220. https://doi.org/10.1017/S0031182003004694

[19] Baneth, G., Florin-Christensen, M., Cardoso, L. and Schnittger, L. (2015) Reclassification of Theileria annae as Babesia vulpes sp. nov. Parasites and Vectors, 8, 207. https://doi.org/10.1186/s13071-015-0830-5

[20] Skuballa, J., Petney, T., Pfäfffie, M. and Taraschewski, H. (2010) Molecular Detection of Anaplasma phagocytophilum in the European Hedgehog (Erinaceus europaeus) and Its Ticks. Vector-Borne and Zoonotic Diseases, 10, 1055-1057. https://doi.org/10.1089/vbz.2009.0150

[21] Estrada-Peña, A. and Jongejan, F. (1999) Ticks Feeding on Humans: A Review of Records on Human-Biting Ixodoidea with Special Reference to Pathogen Transmission. Experimental and Applied Acarology, 23, 685-715. https://doi.org/10.1023/A:1006241108739

[22] Dumont, P., Blair, J., Fourie, J.J., Chester, S.T. and Larsen, D.L. (2014) Evaluation of the Efficacy of Afoxolaner against Two European Dog Tick Species: Dermacentor reticulatus and Ixodes ricinus. Veterinary Parasitology, 20, 216-219. https://doi.org/10.1016/j.vetpar.2014.02.017

[23] Mitchell, E.B., Dorrl, P., Everett, W.R., Chester, S.T. and Larsen, D.L. (2014) Efficacy of Afoxolaner against Dermacentor variabilis Ticks in Dogs. Veterinary Parasitology, 201, 220-222. https://doi.org/10.1016/j.vetpar.2014.02.016

[24] Mitchell, E.B., McCall, J.W., Chester, S.T. and Larsen, D.L. (2014) Efficacy of Afoxolaner against Ixodes scapularis Ticks in Dogs. Veterinary Parasitology, 201, 223-225. https://doi.org/10.1016/j.vetpar.2014.02.015

[25] Kunkle, B., Daly, S., Dumont, P., Drag, M. and Larsen, D.L. (2014) Assessment of the Efficacy of Orally Administered Afoxolaner against Rhipicephalus sanguineus Sensu Lato. Veterinary Parasitology, 20, 226-228. https://doi.org/10.1016/j.vetpar.2014.02.018

[26] Kondo, Y., Kinoshita, G., Drag, M., Chester, S.T. and Larsen, D.L. (2014) Evaluation of the Efficacy of Afoxolaner against Haemaphysalis longicornis on Dogs. Veterinary Parasitology, 201, 229-231. https://doi.org/10.1016/j.vetpar.2014.02.019 
[27] Knaus, M., Rehbein, S., Blair, J., Richard-Mazet, A., Visser, M., Dollhofer, D., Kley, K., Lebon, W., Anderson, A., Larsen, D.L. and Jeannin, P. (2015) Field Efficacy against Tick and Flea Infestations and Safety of Afoxolaner plus Milbemycin Oxime Chewables (NexGard Spectra ${ }^{\circ}$, Merial) in Domestic Dogs in Europe. 25th International Conference of the World Association for the Advancement of Veterinary Parasitology ( $W A A V P)$, Liverpool, 16-20 August 2015, 507.

[28] Cheesman, T., Gogolewski, R., Yoon, S. and Larsen, D.L. (2015) Efficacy of Afoxolaner Chewables (NexGard ${ }^{\circ}$, Merial) and Afoxolaner plus Milbemycin Oxime Chewables (NexGard Spectra', Merial) against the Australian Paralysis Tick, Ixodes holocyclus, in Dogs. $25^{\text {th }}$ International Conference of the World Association for the Advancement of Veterinary Parasitology (WAAVP), Liverpool, 16-20 August 2015, 513.

[29] Rehbein, S., Fourie, J.J., de Vos, C., Anderson, A., Larsen, D.L. and Jeannin, P. (2016) Efficacy of Oral Afoxolaner plus Milbemycin Oxime Chewables against Induced Infestations with Dermacentor reticulatus in Dogs. Parasitology Research, 115, 1845-1851. https://doi.org/10.1007/s00436-016-4924-6

[30] Marchiondo, A.A., Holdsworth, P.A., Fourie, L.J., Rugg, D., Hellmann, K., Snyder, D.E. and Dryden, M.W. (2013) World Association for the Advancement of Veterinary Parasitology (W.A.A.V.P.) Second Edition: Guidelines for Evaluating the Efficacy of Parasiticides for the Treatment, Prevention and Control of Flea and Tick Infestations on Dogs and Cats. Veterinary Parasitology, 194, 84-97. https://doi.org/10.1016/j.vetpar.2013.02.003

[31] Snyder, D.E. and Wiseman, S. (2012) Dose Confirmation and Non-Interference Evaluations of the Oral Efficacy of a Combination of Milbemycin Oxime and Spinosad against the Dose Limiting Parasites, Adult Cat Flea (Ctenocephalides felis) and Hookworm (Ancylostoma caninum), in Dogs. Veterinary Parasitology, 184, 284-290. https://doi.org/10.1016/j.vetpar.2011.11.067

[32] European Medicines Agency (2014) Committee for Medicinal Products for Veterinary Use (CVMP). Assessment Report for NexGard, EMEA/V/C/002729/0000, EMA/18910/2014, London, 21 p.

[33] European Medicines Agency (2015) Committee for Medicinal Products for Veterinary Use (CVMP). Assessment Report for NexGard Spectra, EMEA/V/C/003842/0000, EMA/695949/2014, London, 27 p. 